November 4, 2018

OITS-692

\title{
Charged Higgs decays in models with singlet neutrino in large extra dimensions 1
}

\author{
K. Agashe \&, N.G. Deshpande [?, G.-H. Wu t \\ Institute of Theoretical Science \\ 5203 University of Oregon \\ Eugene OR 97403-5203
}

\begin{abstract}
In models with large extra dimensions and $\mathrm{TeV}$ scale quantum gravity, small (Dirac) neutrino masses can be obtained naturally if the right-handed neutrino propagates in the extra dimensions. In this scenario, with 2 Higgs doublets, we show that the decay of charged Higgs into left-handed charged lepton (say $\tau$ ) and right-handed neutrino can be significantly enhanced, with $O(1)$ branching ratio, due to the large number of Kaluza-Klein states of the right-handed neutrino. Since $\tau$ 's from the standard decay of charged Higgs are right-handed, the above novel charged Higgs decay can provide a distinctive signature of these models at hadron/lepton colliders. Similarly, top quark decays to $b \mu^{+} \nu, b \tau^{+} \nu$ through virtual charged Higgs can be enhanced.
\end{abstract}

\footnotetext{
${ }^{1}$ This work is supported by DOE Grant DE-FG03-96ER40969.

${ }^{2}$ email: agashe@oregon.uoregon.edu

${ }^{3}$ email: desh@oregon.uoregon.edu

${ }^{4}$ email:wu@dirac.uoregon.edu
} 
The framework of large extra dimensions and TeV scale quantum gravity [1] has been extensively studied over the last two years. The idea is that there are $\delta$ additional spatial dimensions of size $R$ in which gravity lives whereas the SM particles are confined to the usual 4 dimensions $(4 D)$. The effective $4 D$ Planck scale, $M_{P l} \sim 2.4 \times 10^{18} \mathrm{GeV}$, is related to the $(4+\delta) D$ "fundamental" Planck scale, $M_{*}$, by

$$
M_{P l}^{2} \sim R^{\delta} M_{*}^{\delta+2} .
$$

Thus, if the extra dimensions are large $\left(R \gg M_{*}^{-1}\right)$, then it is possible that $M_{*} \sim \mathrm{TeV}$ so that the ultraviolet (UV) cut-off for the quantum corrections to the Higgs boson (mass) ${ }^{2}$ is also $\sim$ $\mathrm{TeV}$. Therefore, the gauge hierarchy problem is "solved". The size of the extra dimensions can be as large as $\sim \mathrm{mm}$ for $\delta=2$.

References [2, 3] showed how small neutrino masses can naturally be obtained in this framework even though there is no fundamental mass scale much larger than the weak scale to implement the usual seesaw mechanism for neutrino masses. Since the right-handed (RH) neutrino is a singlet under the SM gauge group, it can live in the extra dimensions [4, 5, 6, 0, 河. In this paper, we study charged Higgs decays to $\tau \bar{\nu}$ in this scenario. For simplicity, consider the case of a single extra dimension labeled by $y$ ( $x$ labels the usual $4 D$ ). A massless Dirac fermion $N$ which is a singlet lives in $5 D$. The $\Gamma$ matrices in $5 D$ can be written as

$$
\Gamma^{\mu}=\left(\begin{array}{cc}
0 & \sigma^{\mu} \\
\bar{\sigma}^{\mu} & 0
\end{array}\right), \Gamma^{5}=\left(\begin{array}{cc}
i & 0 \\
0 & -i
\end{array}\right) .
$$

The Dirac spinor $N$ in the Weyl basis can be written as

$$
N=\left(\begin{array}{l}
\psi \\
\bar{\chi}
\end{array}\right),
$$

where $\psi$ and $\chi$ are 2-component complex spinors (with mass dimension 2). The $5 D$ kinetic term for $N$ is

$$
S_{\text {free }}=\int d^{4} x d y \bar{N}\left(\Gamma^{\mu} \partial_{\mu}+\Gamma^{5} \partial_{y}\right) N .
$$

In the effective $4 D$ theory, $N$ appears as a tower of Kaluza-Klein (KK) states:

$$
\psi=\sum_{n} \frac{1}{\sqrt{R}} \psi^{(n)}(x) e^{i n y / R},
$$

where $\psi^{(n)}$ are $4 D$ states. Similarly, $\chi$ has a KK tower, $\chi^{(n)}$. Thus, in $4 D$, Eq. (四) becomes

$$
S_{\text {free }}=\int d^{4} x \sum_{n}\left[\bar{\psi}^{(n)} \bar{\sigma}^{\mu} \psi^{(n)}+\bar{\chi}^{(n)} \bar{\sigma}^{\mu} \chi^{(n)}+\left(\frac{n}{R} \psi^{(n)} \chi^{(n)}+\text { h.c. }\right)\right] \text {, }
$$

where $n / R$ is the Dirac mass for the KK states. If we assign $N$ the opposite lepton number from the usual lepton doublet $l=(\nu, e)$ (which of course lives on a "3-brane" localized at say $y=0$ ), 
then the interaction between $l$ and $N$ which conserves lepton number is

$$
S_{\text {int }}=\int d^{4} x \frac{\lambda}{\sqrt{M_{*}}} l(x) h^{*}(x) \psi(x, y=0),
$$

where $h$ is the SM Higgs doublet (with hypercharge $-1 / 2$ ) and $\lambda$ is dimensionless.

In the effective $4 D$ theory, Eq. (77) becomes

$$
S_{\text {int }}=\int d^{4} x \sum_{n} \frac{\lambda}{\sqrt{R M_{*}}} l(x) h^{*}(x) \psi^{(n)}(x) .
$$

Using Eq. (11) we get

$$
S_{\text {int }}=\int d^{4} x \sum_{n} \lambda \frac{M_{*}}{M_{P l}} l(x) h^{*}(x) \psi^{(n)}(x) .
$$

This can be easily generalized to the case of $\delta$ extra dimensions resulting in the same effective $4 D$ coupling (for now, we assume that the singlet neutrino lives in the same $\delta$ extra dimensions as the graviton). Thus, we see that the neutrino Yukawa coupling is suppressed by volume of extra dimensions so that the Dirac mass for the SM neutrino is

$$
m=\frac{\lambda}{\sqrt{2}} \frac{M_{*}}{M_{P l}} v
$$

where $v \approx 246 \mathrm{GeV}$ is the Higgs vev.

From Eqns. (6) and (9), we see that $\chi^{(0)}$ decouples and is exactly massless. The full mass matrix for $\nu, \psi^{(0)}, \psi^{(n)}$ and $\chi^{(n)}(n=. .,-2,-1,1,2, .$.$) in the case of one extra dimension is$

$$
\mathcal{L}_{\text {mass }}=\nu_{+} M \nu_{-}^{T}
$$

with

$$
M=\left(\begin{array}{ccccc}
m & m & m & m & \ldots \\
0 & 1 / R & 0 & 0 & \ldots \\
0 & 0 & -1 / R & 0 & \ldots \\
0 & 0 & 0 & 2 / R & \ldots \\
\ldots & \ldots & \ldots & \ldots & \ldots
\end{array}\right)
$$

where

$$
\nu_{+}=\left(\nu, \chi^{(1)}, \chi^{(-1)}, \chi^{(2)}, \ldots\right)
$$

and

$$
\nu_{-}=\left(\psi^{(0)}, \psi^{(1)}, \psi^{(-1)}, \psi^{(2)}, \ldots\right) .
$$

In the limit $m \ll 1 / R$, we have to a good approximation a Dirac fermion $\left(\nu, \psi^{(0)}\right)$ with mass $m$ (see, however, Eq. (19)) and Dirac fermions $\left(\psi^{(n)}, \chi^{(n)}\right)(n=. .,-2,-1,1,2, .$.$) , with masses$ $n / R$ with the mixing between $\nu$ and $\chi^{(n)}$ given by $\sim m R / n \ll 1$.

Since we will study charged Higgs decays to $\tau \bar{\nu}$, we are interested in the case $m^{2} \sim \Delta m_{\text {atm }}^{2} \sim$ $10^{-3}-10^{-2}(\mathrm{eV})^{2}$ (or larger, see later) as indicated by atmospheric neutrino oscillations [9]. For 
$\delta=2$, even for $M_{*} \sim 10 \mathrm{TeV}, 1 / R$ is quite small $\sim 0.01-0.1 \mathrm{eV}$ so that with $m^{2} \sim \Delta m_{\text {atm }}^{2}$, we get $m R \sim 1$, i.e., the above approximation is no longer valid since there is $O(1)$ mixing between the SM neutrino and the light KK neutrinos. Also, due to this large mixing, this scenario might be ruled out by the success of standard theory of Big Bang Nucleosynthesis (BBN) since too many singlet neutrinos will be thermal during nucleosynthesis in the early universe [2, 5, 7]. Hence, in this paper, we will mostly consider $\delta \geq 3$ for which $1 / R \gg 0.1 \mathrm{eV}$. Also, in the supernova 1987a core, neutrinos (of all flavors) undergo coherent oscillations to KK neutrinos leading to energy loss at an unacceptable rate if $m^{2} \gtrsim 10^{-3}(\mathrm{eV})^{2}$ and $1 / R \lesssim 10 \mathrm{keV}[7]$. We will show that the effect of $\mathrm{RH}$ neutrino in extra dimensions on charged Higgs decays can be significant even in the parameter space for which this constraint is satisfied.

To generalize the above to the case of three SM neutrinos, we can add three singlets $N_{i}$ $(i=1,2,3)$.

\section{Charged Higgs decays}

From Eq. (9), it is clear that the neutral Higgs has a coupling $\lambda M_{*} / M_{P l}$ to each of the RH neutrino KK states, $\psi^{(n)}$, so that the neutral Higgs decay to neutrinos can be enhanced by the large number of KK states [2, 10].

Similarly, in a 2-Higgs-doublet model, the charged Higgs decay to (say) $\tau_{L}$ and $(\mathrm{RH})$ neutrino can be enhanced. For simplicity, we assume (as, for example, in a supersymmetric extension of the SM) that the Higgs doublet with hypercharge $-1 / 2$ (denoted by $H_{1}$ ) couples only to $\mathrm{RH}$ up-type quarks and neutrinos (i.e., gives mass to up-type quarks and neutrinos) whereas the hypercharge $+1 / 2$ doublet (denoted by $H_{2}$ ) couples only to $\mathrm{RH}$ charged leptons and down-type quarks - we will refer to this model (in 4D) as 2-Higgs-Doublet Model II (2HDM-II, for short). The ratio of the vev's of $H_{1}$ and $H_{2}$ is denoted by $\tan \beta$ as usual. Neglecting the $\tau$ mass in the phase space integral and also neglecting terms suppressed by $\sim m_{\tau}^{2} / m_{H}^{2}$ in the matrix element, the decay width to LH $\tau$ is given by

$$
\begin{aligned}
\Gamma\left(H^{-} \rightarrow \tau_{L} \psi\right) & \approx \frac{m_{H}}{8 \pi}\left(\frac{m}{v}\right)^{2} \cot ^{2} \beta\left(m_{H} R\right)^{\delta} x_{\delta} \\
& \approx \frac{m_{H}}{8 \pi}\left(\frac{m}{v}\right)^{2} \cot ^{2} \beta\left(\frac{m_{H}}{M_{*}}\right)^{\delta}\left(\frac{M_{P l}}{M_{*}}\right)^{2} x_{\delta},
\end{aligned}
$$

where the factor $\left(m_{H} R\right)^{\delta}$ counts (roughly) the number of RH neutrino KK states lighter than the charged Higgs and we have used Eq. (1) in the second line. We have replaced the sum over KK states by an integral, $\sum_{n} \rightarrow S_{\delta-1} n^{\delta-1} d n$ (where $S_{\delta-1}=2 \pi^{\delta / 2} / \Gamma(\delta / 2)$ is the surface area of a unit-radius sphere in $\delta$ dimensions). [ This together with the phase space integral results in

\footnotetext{
${ }^{5}$ For $\delta$ extra dimensions, $n$ is really $\sqrt{\sum_{i=1}^{\delta} n_{i}^{2}}$, where $n_{i}$ is the momentum (in units of $\sim 1 / R$ ) in the $i^{\text {th }}$ extra dimension.
} 
the factor $x_{\delta}$ which is given by

$$
x_{\delta} \approx \frac{2 \pi^{\delta / 2}}{\Gamma(\delta / 2)}\left(\frac{1}{\delta}-\frac{2}{\delta+2}+\frac{1}{\delta+4}\right) .
$$

As usual the charged Higgs also decays to RH $\tau$ through the $\tau$ Yukawa coupling:

$$
\begin{aligned}
\Gamma\left(H^{-} \rightarrow \tau_{R} \bar{\nu}\right) \approx & {\left[\frac{m_{H}}{8 \pi}\left(\frac{m_{\tau}}{v}\right)^{2} \tan ^{2} \beta\right] } \\
& \times \frac{1}{N^{2}}\left[1+\frac{m^{2}}{M_{*}^{2}} \frac{m_{H}^{\delta-2}}{M_{*}^{\delta-2}} \frac{M_{P l}^{2}}{M_{*}^{2}} \frac{2 \pi^{\delta / 2}}{\Gamma(\delta / 2)}\left(\frac{1}{\delta-2}-\frac{2}{\delta}+\frac{1}{\delta+2}\right)\right] .
\end{aligned}
$$

This decay is also affected by the presence of the RH neutrino in extra dimensions as follows. The SM neutrino (weak eigenstate) is dominantly the lightest neutrino with mass $\sim m$, but it has a small mixture $(\sim m R / n)$ of the heavier neutrinos (see Eq. (12)). This mixing introduces a "normalization factor" $N$ (in the second line of the above equation) given by

$$
N^{2} \approx 1+\sum_{n}\left(\frac{m R}{n}\right)^{2} \approx 1+\frac{m^{2}}{M_{*}^{2}} \frac{M_{P l}^{2}}{M_{*}^{2}} \frac{2 \pi^{\delta / 2}}{\Gamma(\delta / 2)} \frac{1}{\delta-2},
$$

where the sum over KK states is up to the UV cut-off, $M_{*}$. Also, due to this mixing, the charged Higgs also decays into $\mathrm{RH} \tau$ and the heavier neutrinos which have mass $\sim n / R$ and hence a different phase space (compared to the standard decay) - this accounts for the extra factor in the numerator in the second line of Eq. (17) (here the KK states are summed up to the threshold of the decay). For $\delta=2$, we have to replace $1 /(\delta-2)$ by $\ln \left(m_{H} M_{P l} / M_{*}^{2}\right)$ and $\ln \left(M_{P l} / M_{*}\right)$ in the numerator and the denominator, respectively.

It is clear from Eqns. (17) and (18) that the decay width to $\mathrm{RH} \tau$ is actually reduced (as long as $m_{H}<M_{*}$ ) compared to that in 2HDM-II: the term in the first line of Eq. (17) is the decay width in $2 \mathrm{HDM}-\mathrm{II}$.

Also, the mass of the lightest neutrino is modified due to the normalization factor [2]:

$$
m_{\nu} \approx \frac{m}{N} \approx \frac{m}{\sqrt{1+\frac{m^{2}}{M_{*}^{2}} \frac{M_{P l}^{2}}{M_{*}^{2}} \frac{2 \pi^{\delta / 2}}{\Gamma(\delta / 2)} \frac{1}{\delta-2}} .}
$$

Thus, the physical neutrino mass cannot be increased arbitrarily by increasing $m$ (or, in other words, by increasing $\lambda$ ): the upper limit (for given $M_{*}$ and $\delta$ ) is

$$
m_{\nu}^{\max } \approx \frac{M_{*}^{2}}{M_{P l}} \sqrt{\frac{\Gamma(\delta / 2)(\delta-2)}{2 \pi^{\delta / 2}}},
$$

where as before, for $\delta=2, \delta-2$ is replaced by $1 / \ln \left(M_{P l} / M_{*}\right)$. Using Eqs. (18), (19) and (20), we get the useful relations

$$
N^{2} \approx 1+\left(\frac{m}{m_{\nu}^{\max }}\right)^{2}
$$


and

$$
m_{\nu} \approx \frac{m}{\sqrt{1+\left(\frac{m}{m_{\nu}^{\max }}\right)^{2}}} .
$$

The ratio of decay widths to $\mathrm{LH}$ and $\mathrm{RH} \tau$ 's is

$$
x_{L R} \equiv \frac{\Gamma\left(H^{-} \rightarrow \tau_{L} \psi\right)}{\Gamma\left(H^{-} \rightarrow \tau_{R} \bar{\nu}\right)} \sim \cot ^{4} \beta\left(\frac{m}{m_{\tau}}\right)^{2}\left(\frac{m_{H}}{M_{*}}\right)^{\delta}\left(\frac{M_{P l}}{M_{*}}\right)^{2}
$$

where, for simplicity, we have dropped the normalization and phase space factors. For the parameter values $m_{\nu}^{2} \sim 10^{-2}(\mathrm{eV})^{2}$ (as applicable to solutions to the atmospheric neutrino anomaly), $M_{*} \sim 2 \mathrm{TeV}, \delta=3, \tan \beta \sim 2$ and $m_{H} \sim 200 \mathrm{GeV}$, we get $x_{L R} \sim 2 \times 10^{5}$. 回 We see that the decay to LH $\tau$ can dominate over the decay to $\mathrm{RH} \tau$ : the Yukawa coupling to $\tau_{L}$ and an individual KK neutrino is tiny, but there is a large multiplicity factor. It should be possible to measure the $\tau$ polarization at hadron/lepton colliders and thus these two decays can be distinguished.

If the charged Higgs is lighter than the top quark ฤ, then the only other significant decay mode is $\bar{c} s$ which is at most comparable to $\tau_{R} \bar{\nu}$ (and is unaffected by the presence of singlet neutrino in extra dimensions). In this case, since the decay width to LH $\tau \propto m_{H}^{\delta}, x_{L R}$ is a bit smaller than the above estimate, but it can still be $\gg 1$. Then, we see that the BR for the decay mode $\tau_{L} \psi$ can be close to 1 .

If the charged Higgs is heavier than the top quark, then the decay mode $\bar{t} b$ (and maybe $\left.W^{-} h^{0}\right)$ is kinematically allowed. The decay width $H^{-} \rightarrow \bar{t} b$ is

$$
\begin{aligned}
\Gamma\left(H^{-} \rightarrow \bar{t} b\right) \approx & 3 \frac{m_{H}}{8 \pi}\left[\left[\left(\frac{m_{t}}{v}\right)^{2} \cot ^{2} \beta+\left(\frac{m_{b}}{v}\right)^{2} \tan ^{2} \beta\right]\left(1-x_{t}^{2}-x_{b}^{2}\right)-\frac{4 m_{t}^{2} m_{b}^{2}}{v^{2} m_{H}^{2}}\right] \\
& \times \sqrt{\left[1-\left(x_{t}+x_{b}\right)^{2}\right]\left[1-\left(x_{t}-x_{b}\right)^{2}\right]}
\end{aligned}
$$

where the factor of 3 is for the number of colors and $x_{t} \equiv m_{t} / m_{H}, x_{b} \equiv m_{b} / m_{H}$.

The decay width $H^{-} \rightarrow W^{-} h^{0}$ is given by

$$
\Gamma\left(H^{-} \rightarrow W^{-} h^{0}\right) \approx \frac{1}{2 \pi} \cos ^{2}(\beta-\alpha) \frac{p_{W}^{3}}{v^{2}},
$$

where $\alpha$ is the mixing angle of the CP-even neutral Higgs scalars and $p_{W}$ is the magnitude of the 3-momentum of the $W$ boson in the rest frame of the charged Higgs:

$$
p_{W}=\frac{1}{2 m_{H}} \sqrt{\left[m_{H}^{2}-\left(m_{W}+m_{h}\right)^{2}\right]\left[m_{H}^{2}-\left(m_{W}-m_{h}\right)^{2}\right]} .
$$

\footnotetext{
${ }^{6}$ For these values of the parameters, the decay width to $\tau_{R}$ is suppressed by a factor $\sim 10$ relative to 2 HDM-II.

${ }^{7}$ The LEP2 limit on the charged Higgs mass is $\sim 80 \mathrm{GeV}$ [11] and for $\tan \beta \sim 100$, charged Higgs masses up to $\sim 120 \mathrm{GeV}$ are excluded by $\mathrm{CDF}[12$.
} 
Thus, for small $\tan \beta$ and dropping the phase space factors, the ratio of decay widths $H^{-} \rightarrow$ $\tau_{L} \psi$ and $H^{-} \rightarrow \bar{t} b$ is

$$
\frac{\Gamma\left(H^{-} \rightarrow \tau_{L} \psi\right)}{\Gamma\left(H^{-} \rightarrow \bar{t} b\right)} \sim \frac{1}{3}\left(\frac{m}{m_{t}}\right)^{2}\left(\frac{m_{H}}{M_{*}}\right)^{\delta}\left(\frac{M_{P l}}{M_{*}}\right)^{2}
$$

from which we see that even if $H^{-}$is heavier than the top quark, the decay to $\tau_{L} \psi$ can dominate. For the above values of parameters the ratio is $O(100)$ so that the $\mathrm{BR}$ for $H^{-} \rightarrow \tau_{L} \psi$ can be $O(1)$.

\section{Numerical results}

The charged Higgs signatures for the scenario of singlet neutrino in large extra dimensions depend on the parameters $M_{*}, m^{2}, m_{H}, \tan \beta$ and $\delta$ as follows.

First of all, using Eq. (20), we see that for $M_{*} \approx 20 \mathrm{TeV}$, we get $\left(m_{\nu}^{\max }\right)^{2} \approx 3 \times 10^{-3}(\mathrm{eV})^{2}$ (roughly independent of $\delta$ ). Therefore, to get $m_{\nu}^{2} \sim \Delta m_{\text {atm }}^{2} \sim 10^{-3}-10^{-2}(\mathrm{eV})^{2}$, we require $M_{*} \gtrsim 20 \mathrm{TeV}$. Conversely, if $M_{*} \sim$ (a few) $\mathrm{TeV}$, then $m_{\nu}^{2} \ll \Delta m_{\text {atm }}^{2}$ (irrespective of the value of $m$ ). So, we consider three cases: (i) $M_{*} \sim 20 \mathrm{TeV}$, (ii) $M_{*} \gg 10 \mathrm{TeV}$ and (iii) $M_{*} \sim$ (a few) $\mathrm{TeV}$.

Case (i) $M_{*} \sim 20 \mathrm{TeV}$ so that $m_{\nu}^{\max } \sim \sqrt{\Delta m_{\text {atm }}^{2}}$

(a) $m \sim \sqrt{\Delta m_{\text {atm }}^{2}}$ so that $N^{2} \sim O\left(1-10\right.$ ) (see Eq. (21)) and $m_{\nu} \sim \sqrt{\Delta m_{\text {atm }}^{2}}$ (see Eq. (22)). In Fig.1, we plot the BR for $H^{-} \rightarrow \tau \bar{\nu}$ and the polarization of $\tau$ in the $\tan \beta-m_{H}$ plane 9 as a typical example of this case: $\delta=3, m \approx 0.1 \mathrm{eV}$ and $M_{*}=20 \mathrm{TeV}$. This gives $N^{2} \approx 5.5$ and $m_{\nu}^{2} \approx 1.8 \times 10^{-3}(\mathrm{eV})^{2}$. The $\tau$ polarization asymmetry is defined as

$$
A_{\tau}=\frac{\Gamma\left(H^{-} \rightarrow \tau_{L} \psi\right)-\Gamma\left(H^{-} \rightarrow \tau_{R} \bar{\nu}\right)}{\Gamma\left(H^{-} \rightarrow \tau_{L} \psi\right)+\Gamma\left(H^{-} \rightarrow \tau_{R} \bar{\nu}\right)} .
$$

In this example, the enhancement of decay width to LH $\tau$ is not so strong (especially for large $\tan \beta$, see Eqs. (23) and (27)) while the decay width to RH $\tau$ is suppressed (as compared to that in a 2 HDM-II) by a factor of $O(1-10)$ (see Eq. (17)). Thus, the total BR to $\tau$ is smaller than that in 2HDM-II. However, for small $\tan \beta$, it is still possible that a significant fraction of $\tau$ 's from Higgs decay are LH. These features can be seen in Fig.1.

(b) $m^{2} \sim(\text { few eV })^{2} \gg \Delta m_{\text {atm }}^{2}$ so that $N^{2} \gg 1$ and $m_{\nu} \approx m_{\nu}^{\max } \sim \sqrt{\Delta m_{\text {atm }}^{2}}$. A typical example of this case is shown in Fig.2: $\delta=3, m \approx 3 \mathrm{eV}$ and $M_{*}=20 \mathrm{TeV}$. This gives $N^{2} \approx 4000$ and $m_{\nu}^{2} \approx 2.2 \times 10^{-3}(\mathrm{eV})^{2}$.

As seen in the figure, there is a strong enhancement of the decay width to LH $\tau$ for small $\tan \beta$ so that we get $O(1) \mathrm{BR}$ to $\tau$, much larger than in the $2 \mathrm{HDM}-\mathrm{II}$. Also, the decay width to $\mathrm{RH} \tau$ is strongly suppressed $\mathrm{f}$ so that the $\tau$ polarization is dominantly LH.

\footnotetext{
${ }^{8}$ This is the result of the full computation, Eqns. (15), (17), (24) and (25).

${ }^{9}$ It is clear from Eq. (17) that the decay width to $\mathrm{RH} \tau$ is suppressed by the smaller of the two factors $N^{2}$ and $\left(M_{*} / m_{H}\right)^{\delta-2}$, which in this example is $\left(M_{*} / m_{H}\right)^{\delta-2} \sim 100$.
} 


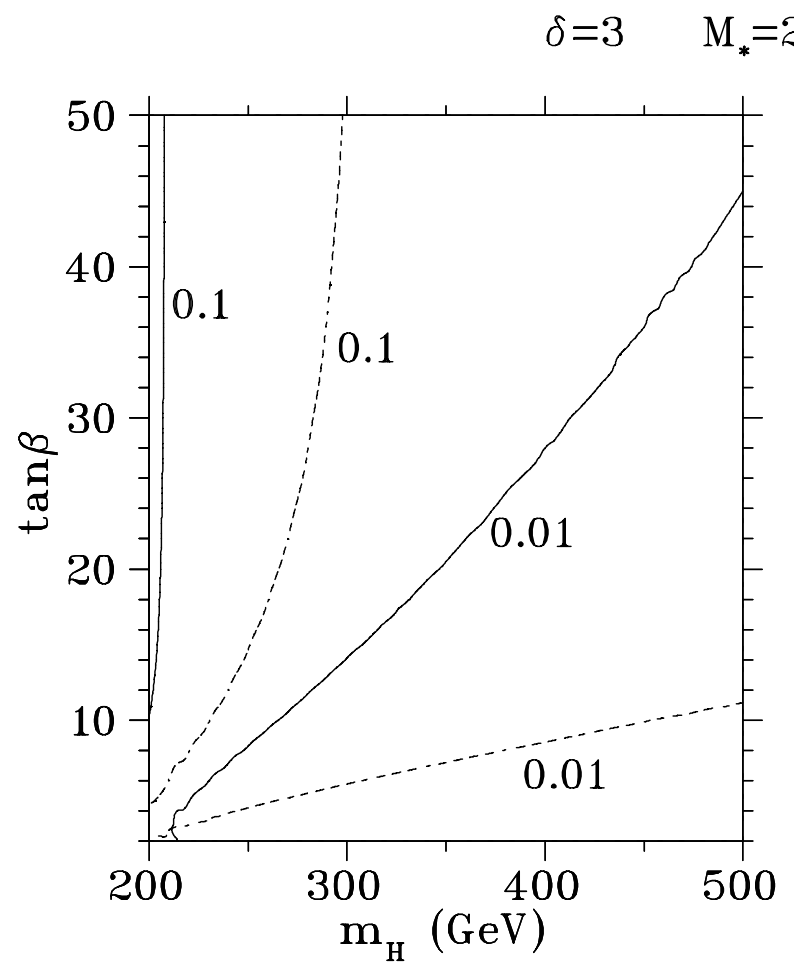

(a) $\mathrm{BR}\left(\mathrm{H}^{-} \rightarrow \tau \nu\right)$

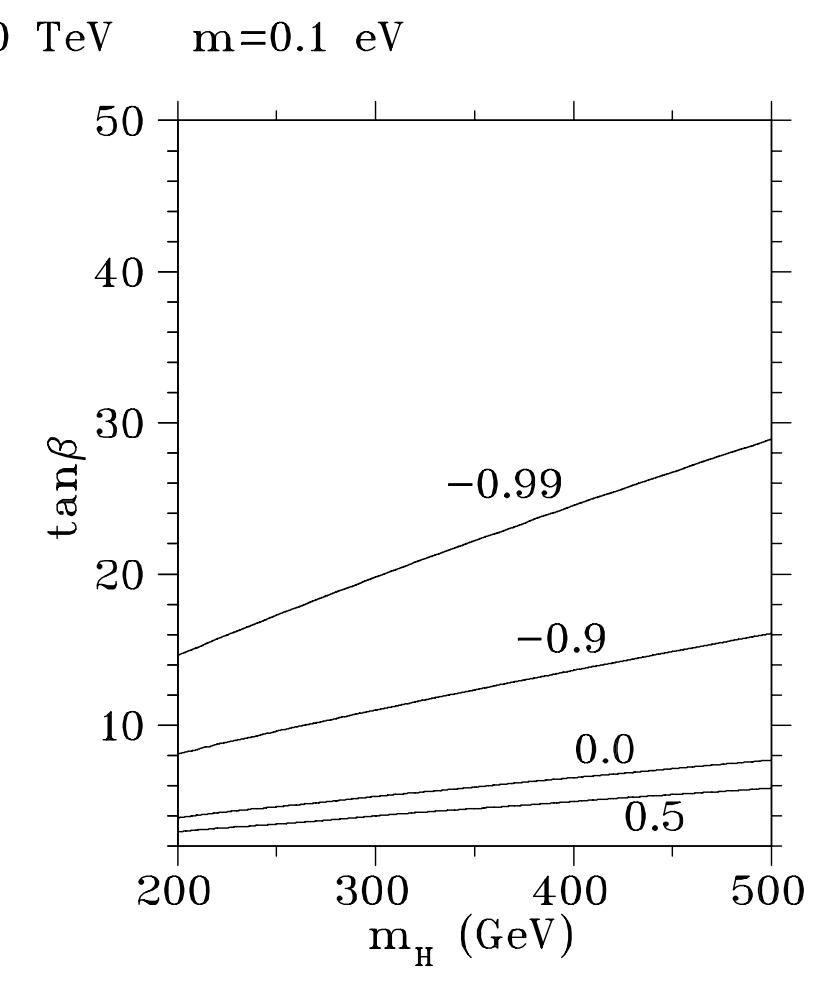

(b) Pol. asymmetry $A_{\tau}$

Figure 1: The contour plot for $B R\left(H^{-} \rightarrow \tau \bar{\nu}\right)$ (solid lines) (left figure) and $\tau$ polarization asymmetry, $A_{\tau}$ (see Eq. (28)) (right figure) in the $m_{H^{-}} \tan \beta$ plane for 3 extra dimensions, $M_{*}=20 \mathrm{TeV}$ and $m \approx 0.1 \mathrm{eV}$ which corresponds to $m_{\nu}^{2} \approx 1.8 \times 10^{-3}(\mathrm{eV})^{2}$ (case (i) (a) of text). The prediction for $B R\left(H^{-} \rightarrow \tau \bar{\nu}\right)$ in a $2 \mathrm{HDM}-\mathrm{II}$ is shown with broken lines (left figure). The decay modes considered for the total charged Higgs decay width are $\tau \bar{\nu}, \bar{t} b$ and $W^{-} h^{0}$. We assume $m_{h^{0}}=110 \mathrm{GeV}$ and the mixing angle of the CP-even neutral Higgs scalars, $\alpha=\pi / 6$. 


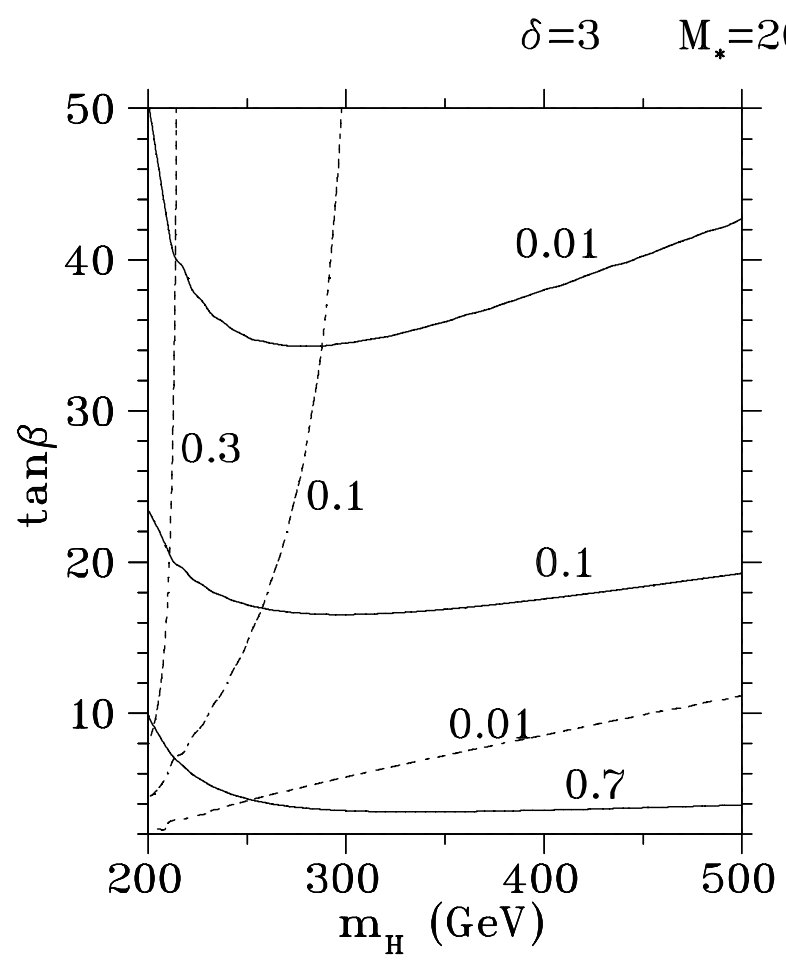

(a) $\mathrm{BR}\left(\mathrm{H}^{-} \rightarrow \tau \nu\right)$

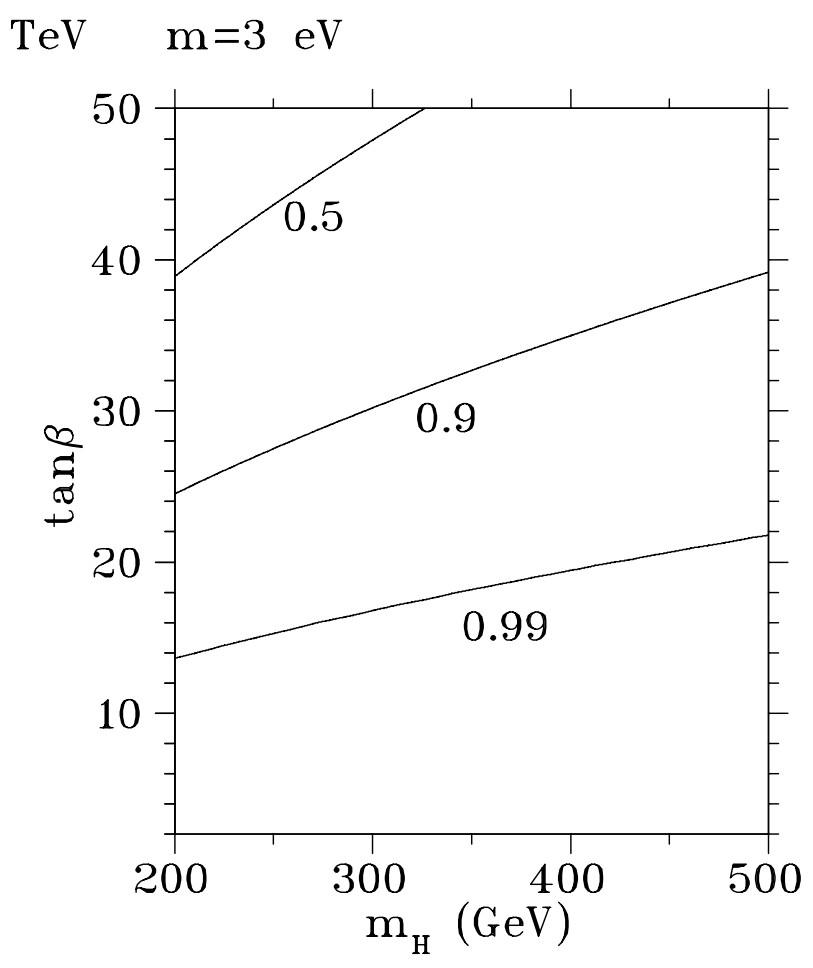

(b) Pol. asymmetry $A_{\tau}$

Figure 2: The contour plot for $B R\left(H^{-} \rightarrow \tau \bar{\nu}\right)$ (solid lines) (left figure) and $\tau$ polarization asymmetry, $A_{\tau}$ (see Eq. (28)) (right figure) in the $m_{H^{-}} \tan \beta$ plane for 3 extra dimensions, $M_{*}=20 \mathrm{TeV}$ and $m \approx 3 \mathrm{eV}$ which corresponds to $m_{\nu}^{2} \approx 2.2 \times 10^{-3}(\mathrm{eV})^{2}$ (case (i) (b) of text). The prediction for $B R\left(H^{-} \rightarrow \tau \bar{\nu}\right)$ in a $2 \mathrm{HDM}-\mathrm{II}$ is shown with broken lines (left figure). The decay modes considered for the total charged Higgs decay width are $\tau \bar{\nu}, \bar{t} b$ and $W^{-} h^{0}$. We assume $m_{h^{0}}=110 \mathrm{GeV}$ and the mixing angle of the CP-even neutral Higgs scalars, $\alpha=\pi / 6$. 
If $\delta>3$, then, for both cases (i) (a) and (b), the decay width to LH $\tau$ is small since it is $\propto\left(m / M_{*}\right)^{2}\left(m_{H} / M_{*}\right)^{\delta}$. On the other hand, in case (i) (a), the decay width to RH $\tau$ is suppressed by $\sim N^{2}$ and thus this suppression remains about the same. In case (i) (b), the decay width to RH $\tau$ is suppressed by $\sim\left(M_{*} / m_{H}\right)^{\delta-2}$ and so the suppression becomes stronger.

A few comments on the case (i) (b) are in order. It is clear that, for a given value of $M_{*}$ and $\delta$, this effect occurs only for a specific value of the physical neutrino mass, i.e, $m_{\nu} \approx m_{\nu}^{\max }$. Also, $m \sim$ (few) $\mathrm{eV}$ and $M_{*} \sim 20 \mathrm{TeV}$ implies that the dimensionless $(5 D)$ coupling, $\lambda$, is much larger than 1 (see Eq. (10)). However, from the point of view of the effective $4 D$ theory, the Yukawa coupling is $\lambda M_{*} / M_{P l}$, which is much smaller than 1 so that the $4 D$ theory is still perturbative. The $O(m R)^{2}$ correction to the normalization factor (Eq. (18)) is very large and so one might worry about the higher order corrections $\sim \sum_{n}(m R)^{4} / n^{4}$, but these are suppressed by $\sim m^{2} / M_{*}^{2}$ relative to the $O(m R)^{2}$ corrections and hence can be neglected.

For $M_{*} \sim 20 \mathrm{TeV}$ and $\delta \geq 3$, we get $1 / R \gtrsim 10 \mathrm{keV}$ so that the SN1987a constraint is satisfied, although the $\delta=3$ case is marginal.

Case (ii) $M_{*} \gg 10 \mathrm{TeV}$ (decoupling limit)

In this case we have $\left(m_{\nu}^{\max }\right)^{2} \gg \Delta m_{\text {atm }}^{2}$ (roughly independent of $\delta$ ) so that, to get $m_{\nu} \sim$ $\sqrt{\Delta m_{\text {atm }}^{2}}$, we require $m \sim \sqrt{\Delta m_{\text {atm }}^{2}}$. As a result, the decay width to LH $\tau$ is negligible since it is $\propto\left(m / M_{*}\right)^{2}\left(m_{H} / M_{*}\right)^{\delta}$. Also, $N^{2} \approx 1$ and so BR to RH $\tau$ is about the same as in 2HDM-II. Thus we recover the 2HDM-II predictions in this case.

Of course, in the case of degenerate neutrinos, $m_{\nu_{\mu}} \approx m_{\nu_{\tau}}$, it is possible that $m_{\nu_{\tau}}^{2} \gg \Delta m_{\mathrm{atm}}^{2}$, with $\left|m_{\nu_{\tau}}^{2}-m_{\nu_{\mu}}^{2}\right| \sim \Delta m_{\mathrm{atm}}^{2}$ to account for the atmospheric neutrino anomaly. For example, one can choose $M_{*} \sim 100 \mathrm{TeV}$ and $m$ (for $\nu_{\tau}$ ) $\sim \mathrm{eV}$ or larger. This results in $N^{2} \sim O(1-10)$ or $N^{2} \gg 1, m_{\nu_{\tau}} \sim m_{\nu}{ }^{\max } \sim \mathrm{eV}$ and a phenomenology similar to case (i) (a) or (b). However, in this case, $m_{\nu_{\mu}}^{2} \sim(\mathrm{eV})^{2}$ also which might be ruled out by the limit from $\mathrm{BR}(\mu \rightarrow e \gamma)$ [ [4] (depending on $\nu_{e}-\nu_{\mu}$ mixing).

For $M_{*} \gg 10 \mathrm{TeV}$ and $\delta \geq 3$, we get $1 / R \gg 10 \mathrm{keV}$ so that the SN1987a constraint is easily satisfied.

Case (iii) $M_{*} \sim$ (few) $\mathrm{TeV}$.

In this case, $\left(m_{\nu}^{\max }\right)^{2} \ll \Delta m_{\text {atm }}^{2}$ and the relevance to atmospheric neutrino oscillations is not clear. Of course, it is possible that $m_{\nu_{\tau}}^{2} \ll m_{\nu_{\mu}}^{2} \sim \Delta m_{\text {atm }}^{2}$ ("inverted hierarchy"), but this scale for $m_{\nu_{\mu}}$ has to be obtained by some other mechanism.

Nevertheless, as discussed earlier, for $m^{2} \sim 10^{-2}(\mathrm{eV})^{2}$, the decay width to LH $\tau$ is very strongly enhanced so that the BR for $H^{-}$decay to left-handed $\tau$ can be $O(1)$ (even for moderate $\tan \beta$ or large $\delta$ ), whereas the decay width to $\mathrm{RH} \tau$ is suppressed compared to $2 \mathrm{HDM}$-II so that the $\tau$ polarization is $\sim 100 \% \mathrm{LH}$. However, for $\delta=3$, we get $1 / R \sim 100 \mathrm{eV}$ and hence the SN1987a bound is violated. Whereas, for $\delta \geq 4$, this constraint is satisfied.

To summarize, we see that the following observations at hadron/lepton colliders can be a 
smoking gun signal for RH neutrino in large extra dimensions:

1. $\tau$ 's from charged Higgs decays are dominantly left-handed as in case (iii): $M_{*} \sim \mathrm{TeV}$, $m^{2} \sim 10^{-3}(\mathrm{eV})^{2}$, case (i) (a) $M_{*} \sim 10 \mathrm{TeV}, m \sim 0.1 \mathrm{eV}$, for small $\tan \beta$, and case (i) (b): $M_{*} \sim 10 \mathrm{TeV}, m \sim \mathrm{eV}$

2. suppressed (relative to 2HDM-II) BR for charged Higgs decay to RH $\tau$ as in case (iii) and case $(\mathrm{i}): M_{*} \sim 10 \mathrm{TeV}$ and $m^{2} \gtrsim 10^{-3}(\mathrm{eV})^{2}$

3. $\operatorname{BR}\left(H^{-} \rightarrow \tau \bar{\nu}\right)$ different than that in $2 \mathrm{HDM}-\mathrm{II}$. In particular, for small $\tan \beta$, this $\mathrm{BR}$ is small in 2HDM-II whereas in cases (i) (b) and (iii), BR $\left(H^{-} \rightarrow \tau \bar{\nu}\right)$ can be $O(1)$.

Of course, the effect is sensitive to the values of $m_{H}, \tan \beta$ and $\delta$.

If $m^{2}$ for $\nu_{e, \mu}$ are of the same order as in the case (i) (b) or (iii), then the charged Higgs decays to LH $e, \mu$ can also be enhanced (depending as usual on the parameter values) - of course, charged Higgs decays to $\mathrm{RH} e, \mu$ are negligible due to the small Yukawa couplings. In fact, the case $M_{*} \sim$ (few) $\mathrm{TeV}$ can give $m_{\nu_{e, \mu}}$ of the correct order for solar neutrino oscillations. However, these scenarios might be constrained (depending on the $\nu_{e}-\nu_{\mu}$ mixing) by the limit on $\operatorname{BR}(\mu \rightarrow e \gamma)[4$.

\section{Singlet neutrino in sub-spaces}

It is also possible that the singlet neutrino lives in smaller number of extra dimensions, $\delta_{\nu}<\delta$, than the graviton [2]. For simplicity, assume that all the extra dimensions are of the same size, $R$. In this case, the effective $4 D$ neutrino Yukawa interaction is

$$
S_{\text {int }}=\int d^{4} x \sum_{n} \frac{\lambda}{\sqrt{\left(R M_{*}\right)^{\delta_{\nu}}}} l(x) h^{*}(x) \psi^{(n)}(x) .
$$

Using Eq. (四) we get

$$
S_{\mathrm{int}}=\int d^{4} x \sum_{n} \lambda\left(\frac{M_{*}}{M_{P l}}\right)^{\delta_{\nu} / \delta} l(x) h^{*}(x) \psi^{(n)}(x) .
$$

Similarly, in the expressions for the charged Higgs decay widths to $\tau_{L}$ (Eqs. (15) and (16)) and $\tau_{R}$ (Eqs. (17) and (18)) and in the relation between $m$ and the physical neutrino mass, $m_{\nu}$ (Eq. (19)), we do the replacements:

$$
\begin{aligned}
\delta & \rightarrow \delta_{\nu} \\
\left(\frac{M_{P l}}{M_{*}}\right)^{2} & \rightarrow\left(\frac{M_{P l}}{M_{*}}\right)^{2\left(\delta_{\nu} / \delta\right)} .
\end{aligned}
$$

In this case, the maximum value of the physical neutrino mass for given $M_{*}, \delta_{\nu}$ and $\delta$ is given by

$$
m_{\nu}^{\max } \approx M_{*}\left(\frac{M_{*}}{M_{P l}}\right)^{\delta_{\nu} / \delta} \sqrt{\frac{\Gamma\left(\delta_{\nu} / 2\right)\left(\delta_{\nu}-2\right)}{2 \pi^{\delta_{\nu} / 2}}}
$$


which is larger than in the case $\delta_{\nu}=\delta$ (Eq. (20)). For example, for $M_{*} \approx 1 \mathrm{TeV}, \delta=6$ and $\delta_{\nu}=5$, we get $m_{\nu}^{\max } \sim \sqrt{\Delta m_{\mathrm{atm}}^{2}}$. Thus, we can get $m_{\nu}^{2} \sim \Delta m_{\mathrm{atm}}^{2}$ even for $M_{*} \sim$ a few $\mathrm{TeV}$ (unlike in the case earlier). For $m \sim \sqrt{\Delta m_{\text {atm }}^{2}}$, we get $N^{2} \sim O(1-10)$ and the charged Higgs decays to $\tau$ are similar to the case (i) (a), whereas for $m \sim \mathrm{eV}$, we get $N^{2} \gg 1$ in which case the charged Higgs phenomenology is similar to the case (i) (b). For smaller values of $\delta_{\nu} / \delta$ (for example, $\left.\delta_{\nu}=3, \delta=4\right)$ and $M_{*} \sim \mathrm{TeV}$, we get $\left(m_{\nu}^{\max }\right)^{2} \gtrsim(\mathrm{eV})^{2} \gg \Delta m_{\text {atm }}^{2}$. Thus, to get large decay width to $\tau_{L}$, we require $m \gtrsim \mathrm{eV}$ which results in $m_{\nu}^{2} \gg \Delta m_{\mathrm{atm}}^{2}$.

Next, we study the effects of virtual charged Higgs in the scenario of RH neutrino in large extra dimensions.

\section{Effects of virtual charged Higgs}

\section{1. $B$ meson decays: $B^{-} \rightarrow l \bar{\nu}$}

The decay width in the SM is

$$
\Gamma_{W}\left(B^{-} \rightarrow l_{R} \bar{\nu}\right) \approx \frac{1}{8 \pi} m_{B} m_{l}^{2} f_{B}^{2}\left|V_{u b}\right|^{2} G_{F}^{2}\left(1-\frac{m_{l}^{2}}{m_{B}^{2}}\right)^{2},
$$

where $i f_{B} p_{\mu}=\left\langle 0\left|\bar{b} \gamma_{\mu} \gamma_{5} u\right| B^{+}(\mathbf{p})\right\rangle$ and $l=e, \mu, \tau$.

In 2HDM-II with RH neutrino in large extra dimensions, the charged Higgs exchange results in a decay width

$$
\Gamma_{H}\left(B^{-} \rightarrow l_{L} \psi\right) \sim \frac{1}{8 \pi} m_{B}^{3}\left(\frac{m m_{b}}{m_{H}^{2}}\right)^{2} f_{B}^{2}\left|V_{u b}\right|^{2} G_{F}^{2}\left(\frac{m_{B}}{M_{*}}\right)^{\delta}\left(\frac{M_{P l}}{M_{*}}\right)^{2},
$$

where the last two factors are from the multiplicity of KK states as usual. There is also a charged Higgs exchange contribution to the decay to $\mathrm{RH} l$ (which is significant only for $\tau$ ), but this is not enhanced by the multiplicity factor and hence is not as important.

However, the larger effect of $\mathrm{RH}$ neutrino living in extra dimensions on the $B^{-} \rightarrow l \bar{\nu}$ decay width is due to modification of the $W$ exchange amplitude as follows. The SM neutrino has a small mixture $\sim m R / n$ of the heavier neutrinos, $\chi^{(n)}$ 's, which have Dirac masses $\sim n / R$ (with the $\psi^{(n)}$ 's) (see Eq. (12)). Due to this effect, the virtual $W$ can decay into LH $l$ and RH neutrino, $\psi^{(n)}$ 's. The same effect in $\pi^{-} \rightarrow e \bar{\nu}, \mu \bar{\nu}$ decays was considered in [6]. The decay width $\Gamma_{W}\left(B^{-} \rightarrow l_{L} \psi\right)$ is (up to phase space factors) independent of the charged lepton mass. The present experimental limit on $\mathrm{BR}\left(B^{-} \rightarrow \tau^{-} \bar{\nu}\right)$ is weaker than that on $\operatorname{BR}\left(B^{-} \rightarrow \mu^{-} \bar{\nu}\right)$. In the case of $\nu_{e}$, the ratio of $\pi^{-} \rightarrow e \bar{\nu}$ to $\pi^{-} \rightarrow \mu \bar{\nu}$ decay widths [6] gives a much stronger constraint on $M_{*}$ than $\mathrm{BR}\left(B^{-} \rightarrow e_{L} \psi\right)$. For $\delta=2$, the effect of $\mathrm{KK}$ neutrinos on the ratio of $\pi^{-} \rightarrow e \bar{\nu}$ to $\pi^{-} \rightarrow \mu \bar{\nu}$ decay widths depends mostly on $m_{\nu_{e}}$ and hence does not constrain the $m_{\nu_{\mu}}$ case as

\footnotetext{
${ }^{10}$ Or more directly, the chirality flip $m$ converts $\nu$ into $\psi^{(n)}$ : see Eq. 12.
} 
much. So, we consider $B^{-} \rightarrow \mu_{L} \psi$ decay for which

$$
\begin{aligned}
\Gamma_{W}\left(B^{-} \rightarrow \mu_{L} \psi\right) & \approx \frac{1}{8 \pi} G_{F}^{2} m_{B} f_{B}^{2}\left|V_{u b}\right|^{2} \sum_{n}\left(\frac{n}{R}\right)^{2}\left(\frac{m R}{n}\right)^{2} \frac{1}{N^{2}}\left(1-\frac{n^{2} / R^{2}}{m_{B}^{2}}\right)^{2} \\
& \approx \frac{1}{8 \pi} G_{F}^{2} m_{B} f_{B}^{2}\left|V_{u b}\right|^{2} m_{\nu}^{2}\left(\frac{m_{B}}{M_{*}}\right)^{\delta}\left(\frac{M_{P l}}{M_{*}}\right)^{2} x_{\delta},
\end{aligned}
$$

where $N^{2}$ is the normalization factor, Eq. (18), and we have used $m_{\nu} \approx m / N$ in the second line. As usual, the factor $x_{\delta}$, Eq. (16), in the second line comes from converting the sum over KK states to an integral and the phase space integral, where we have neglected $m_{\mu}$. Note that this effect depends on the $\mu$ neutrino mass and is independent of $m_{H}$. We can see that this effect is larger than the charged Higgs effect (Eq. (34)) by a factor $\sim\left(m_{H} / m_{B}\right)^{4}$.

The total decay width of the $B$ meson, $\Gamma_{B}$, can be approximated by the decay width for the $b$ quark decay, $b \rightarrow c(\bar{c} s, \bar{u} d, l \bar{\nu})$ :

$$
\Gamma_{B} \approx \frac{1}{192 \pi^{3}}\left|V_{c b}\right|^{2} G_{F}^{2} m_{b}^{5} \times 6,
$$

where we have included a factor of 6 for the number of virtual $W$ decay modes weighted by the phase space.

We use $f_{B} / m_{B} \approx 1 / 20$ and $\left|V_{u b} / V_{c b}\right| \approx 0.1$. Then, using Eqs. (33) and (36), we see that $\mathrm{BR}\left(B^{-} \rightarrow \mu_{R} \bar{\nu}\right) \square$ is smaller than the present experimental limit $\approx 2 \times 10^{-5}$ [13] by a factor of $O(100)$. So, to obtain a constraint on $M_{*}$, we require that decay width $B^{-} \rightarrow \mu_{L} \psi$ (Eq. (35)) result in a BR smaller than the present limit. This gives

$$
\begin{aligned}
\frac{\Gamma_{W}\left(B^{-} \rightarrow \mu_{L} \psi\right)}{\Gamma_{B}} & \approx x \frac{24 \pi^{2}}{6}\left(\frac{f_{B}}{m_{B}}\right)^{2}\left(\frac{\left|V_{u b}\right|}{\left|V_{c b}\right|}\right)^{2}\left(\frac{m_{\nu}}{m_{B}}\right)^{2}\left(\frac{m_{B}}{M_{*}}\right)^{\delta}\left(\frac{M_{P l}}{M_{*}}\right)^{2} \\
& \lesssim 2 \times 10^{-5} .
\end{aligned}
$$

Consider the case $\delta=2$. Using Eq. (20), we see that for $M_{*} \approx 30 \mathrm{TeV}$, we get $\left(m_{\nu}^{\max }\right)^{2} \approx 10^{-3}$ $(\mathrm{eV})^{2} \sim \Delta m_{\mathrm{atm}}^{2}$ so that to get $m_{\nu}^{2} \sim \Delta m_{\mathrm{atm}}^{2}$, we require $M_{*} \gtrsim 30 \mathrm{TeV}$. $\mathrm{Z}$ Then, the above formula shows that $\mathrm{BR}\left(B^{-} \rightarrow \mu_{L} \psi\right)$ is below the present limit by a factor of $\approx 3$. Thus, if the ongoing $B$-physics experiments improve the experimental limit by a factor of $\approx 3$, then the constraint on $M_{*}$ from $\operatorname{BR}\left(B^{-} \rightarrow \mu_{L} \psi\right)$ will become significant. For $\delta \geq 3$, the minimum value of $M_{*}$ required to get $m_{\nu}^{2} \sim \Delta m_{\mathrm{atm}}^{2}$ is a bit smaller, but $\mathrm{BR}\left(B^{-} \rightarrow \mu_{L} \psi\right)$ is suppressed by an extra factor of $\left(m_{B} / M_{*}\right)^{\delta-2}$ so that the current experimental bound is easily satisfied. Whereas for $\delta \geq 3$, the effect of KK neutrinos on the ratio of $\pi^{-} \rightarrow e \bar{\nu}$ to $\pi^{-} \rightarrow \mu \bar{\nu}$ decay widths depends on $\Delta m_{e-\mu}^{2}$ and gives a strong constraint on $M_{*}$ [6].

\footnotetext{
${ }^{11}$ This decay width is actually reduced compared to the SM for the same reason as in the case of $H^{-} \rightarrow \tau_{R} \bar{\nu}$.

${ }^{12}$ For this scale, $1 / R \sim 0.5 \mathrm{eV}$. Thus, if $m^{2} \sim 10^{-3}-10^{-2}(\mathrm{eV})^{2}$, then $m R$ is still smaller than 1 and BBN constraint can also be evaded. However, the SN1987a bound is violated.
} 


\section{Lepton flavor violating decays: $\mu \rightarrow e \gamma$}

There is also a charged Higgs exchange contribution at one-loop to flavor violating decays, for example, $\mu \rightarrow e \gamma$. The one-loop $W$ exchange contribution to this decay with RH neutrino in large extra dimensions was studied in [4]. As usual, this decay is enhanced by the large number of $\mathrm{RH}$ neutrino KK states in the loop - this multiplicity factor compensates for the small neutrino mass (or Yukawa coupling). For $\Delta m_{e-\mu}^{2} \sim 10^{-5}(\mathrm{eV})^{2}$ and $\theta_{e-\mu} \sim \pi / 4$, the limits on $M_{*}$ are $\sim 100 \mathrm{TeV}$ and $\sim 35 \mathrm{TeV}$ for $\delta=2$ and 3, respectively.

The dominant contribution (with a single Higgs doublet) comes from longitudinal $W$ boson and heavy KK states (with masses close to the UV cut-off, $M_{*}$ ) in the loop [⿴囗十 (at least for $\delta \geq 3$ ). Therefore, in 2HDM-II, the contribution with charged Higgs in the loop is of the same order as the $W$ boson exchange effect as long as $m_{H}<$ the cut-off, $M_{*}$.

\section{Top quark decay: $t \rightarrow b \tau^{+} \nu$}

The SM decay $t \rightarrow b W^{+}$has width

$$
\begin{aligned}
\Gamma\left(t \rightarrow b W^{+}\right) & \approx \frac{G_{F} m_{t}^{3}}{8 \pi \sqrt{2}}\left|V_{t b}\right|^{2}\left(1-x_{W}^{2}\right)\left(1+x_{W}^{2}-2 x_{W}^{4}\right) \\
& \approx 0.6 \frac{G_{F} m_{t}^{3}}{8 \pi}
\end{aligned}
$$

where $x_{W} \equiv m_{W} / m_{t} \approx 1 / 2$ and $\left|V_{t b}\right| \approx 1$. The decay width to $b \tau^{+} \nu$ is smaller by a factor of $1 / 9$.

The (anti-)top quark decay to LH $\tau$ with charged Higgs exchange is enhanced by the large number of RH $\nu$ KK states. For small $\tan \beta$, we get:

$$
\Gamma\left(\bar{t} \rightarrow \bar{b} \tau_{L} \psi\right) \sim \frac{\sqrt{2} G_{F} m_{t}^{3}}{192 \pi^{3}}\left(\frac{m}{v}\right)^{2} \cot ^{4} \beta\left(\frac{m_{t}}{m_{H}}\right)^{4}\left(\frac{m_{t}}{M_{*}}\right)^{\delta}\left(\frac{M_{P l}}{M_{*}}\right)^{2} .
$$

Thus, the ratio of the top decay widths to $b \tau^{+} \nu$ due to charged Higgs exchange and that in the $\mathrm{SM}$ is

$$
\sim \frac{9 \sqrt{2}}{0.624 \pi^{2}}\left(\frac{m}{v}\right)^{2} \cot ^{4} \beta\left(\frac{m_{t}}{m_{H}}\right)^{4}\left(\frac{m_{t}}{M_{*}}\right)^{\delta}\left(\frac{M_{P l}}{M_{*}}\right)^{2}
$$

which is $O(1)$ for $m^{2} \sim 10^{-2}(\mathrm{eV})^{2}, \tan \beta \sim 2, M_{*} \sim 2 \mathrm{TeV}, \delta=3$ and $m_{H} \sim 200 \mathrm{GeV}$, i.e., the top decay width to $b \tau^{+} \nu$ can be enhanced by $O(100 \%)$. $⿴$ At the LHC, it should be possible to measure the top BR's at the $\sim 10 \%$ level so that the above effect can be observed. If the three SM neutrinos masses are (roughly) of the same order, then the same effect will be observed in top quark decays to $b \mu^{+} \nu$ and $b e^{+} \nu$. On the other hand, if $m_{\nu_{\mu, e}} \ll m_{\nu_{\tau}}$, then the top quark decays to $e^{+}, \mu^{+}$will be unaffected - this violation of lepton universality in top quark decays can be observed at the LHC or even in Run II of the Tevatron.

\footnotetext{
${ }^{13}$ Of course, for $M_{*} \sim 2 \mathrm{TeV}$, the physical neutrino mass is smaller than $\sqrt{\Delta m_{\text {atm }}^{2}}$ and also the SN1987a bound is violated for $\delta=3$.
} 
In summary, we have studied the effects of $\mathrm{RH}$ neutrino living in large extra dimensions on charged Higgs phenomenology. We have shown that in this model charged Higgs decays to lefthanded $\tau$ can be enhanced, with $O(1)$ branching ratio, and decays to $\mathrm{RH} \tau$ can be suppressed. Constraints from SN1987a energy loss indicate that effects of bulk neutrino cannot be observed in atmospheric neutrino oscillations [7]; however, we have seen that the effects in charged Higgs decays can still be observed. Thus, charged Higgs decays can provide signatures for these models.

\section{References}

[1] N. Arkani-Hamed, S. Dimopoulos, G. Dvali, hep-ph/9803315, Phys. Lett. B 429, 263 (1998) and hep-ph/9807344, Phys. Rev. D 59 (1999) 086004; I. Antoniadis et al, hep-ph/9804398, Phys. Lett. B 436 (1998) 257.

[2] N. Arkani-Hamed et al., talk by S. Dimopoulos at SUSY 98, Oxford, July 11-17, 1998 and hep-ph/9811448.

[3] K.R. Dienes, E. Dudas, T. Gherghetta, hep-ph/9811428, Nucl. Phys. B 557 (1999) 25.

[4] A.E. Faraggi, M. Pospelov, hep-ph/9901299, Phys. Lett. B 458 (1999) 237.

[5] G. Dvali, A. Smirnov, hep-ph/9904211, Nucl. Phys. B 563 (1999) 63.

[6] A. Das, O.C.W. Kong, hep-ph/9907272, Phys. Lett. B 470 (1999) 149.

[7] R. Barbieri, P. Creminelli, A. Strumia, hep-ph/0002199.

[8] R.N. Mohapatra, S. Nandi, A. Perez-Lorenzana, hep-ph/9907520, Phys. Lett. B466 (1999) 115; A. Ioannisian, A. Pilaftsis, hep-ph/9907522; G.C. McLaughlin, J.N. Ng, hepph/9909558, Phys. Lett. B470 (1999) 157 and nucl-th/0003023; R.N. Mohapatra, A. PerezLorenzana, hep-ph/9910474, Nucl. Phys. B 576 (2000) 466; A. Lukas, A. Romanino, hep$\mathrm{ph} / 0004130$.

[9] Y. Fukuda et al. (Super-Kamiokande collaboration), hep-ex/9807003, Phys. Rev. Lett. 81 (1998) 1562 and hep-ex/9812014, Phys. Rev. Lett. 82 (1999) 2644.

[10] S.P. Martin, J.D.Wells, hep-ph/9903259, Phys. Rev. D 60 (1999) 035006.

[11] ALEPH collaboration, ALEPH 2000-011, CONF 2000-008, see the website http://alephwww.cern.ch/ALPUB/oldconf/oldconf_00.htm].

[12] T. Affolder et al. (CDF collaboration), hep-ex/9912013, Phys. Rev. D 62 (2000) 012004.

[13] C. Caso et al. (Particle Data Group), Eur. Phys. J. C 3 (1998) 1. 